\title{
Stability of the permanent rotations of an asymmetric gyrostat in a uniform Newtonian field
}

\author{
M. Iñarrea ${ }^{\mathrm{a}}$, V. Lanchares ${ }^{\mathrm{b}, *}$, A. I. Pascual ${ }^{\mathrm{b}}$, A. Elipe $\mathrm{E}^{\mathrm{c}, \mathrm{d}}$ \\ ${ }^{a}$ Área de Física Aplicada. Universidad de La Rioja, 26006 Logroño, Spain. \\ ${ }^{b}$ Dept. Matemáticas y Computación. Universidad de La Rioja, 26004 Logroño, Spain. \\ ${ }^{c}$ Centro Universitario de la Defensa de Zaragoza, 50090 Zaragoza, Spain. \\ ${ }^{d}$ GME-IUMA, Universidad de Zaragoza, 50009 Zaragoza, Spain.
}

\begin{abstract}
The stability of the permanent rotations of a heavy gyrostat is analyzed by means of the Energy-Casimir method. Sufficient and necessary conditions are established for some of the permanent rotations. The geometry of the gyrostat and the value of the gyrostatic moment are relevant in order to get stable permanent rotations. Moreover, the necessary conditions are also sufficient, for some configurations of the gyrostat.
\end{abstract}

Keywords: stability, gyrostat rotation, energy-Casimir method 2000 MSC: 70E55, 34C35, 34D20

\section{Introduction}

A gyrostat $\mathcal{G}$ is a mechanical system made of a rigid body $\mathcal{P}$ called the platform and other bodies $\mathcal{R}$ called the rotors, connected to the platform in such a way that the motion of the rotors does not modify the distribution of mass of the gyrostat $\mathcal{G}$. Due to this double spinning, the platform on the one hand and the rotors on the other, the gyrostat is also known with the name of dual-spin body.

In Astrodynamics, gyrostats play an essential role, since they are used for controlling the attitude dynamics of a spacecraft and for stabilizing their rotations. See, for instance, Cochran [8], Hall [15, 16, 17], Elipe and coworkers $[10,11,12,13,20,26]$, Vera [36], Aslanov [4, 5] and also Hughes [19] for further references.

Besides its practical interest, the rotational motion of a gyrostat is very interesting from a mathematical point of view. Indeed, principal moments of inertia and gyrostatic momenta may be considered as parameters in the Euler

\footnotetext{
* Corresponding author

Email addresses: manuel.inarrea@unirioja.es (M. Iñarrea), vlancha@unirioja.es (V. Lanchares), aipasc@unirioja.es (A. I. Pascual), elipe@unizar.es (A. Elipe)
} 
equations of motion and there is a wide variety of possible equilibria, trajectories and bifurcations even in the simplest case of a gyrostat in free motion, that is to say, under no external forces. The authors have been studying this case for several years, and one of the main results obtained is the proof that when the gyrostat motion is formulated in terms of the angular momentum components, this problem is equivalent to a parametric quadratic Hamiltonian [12], and for those class of quadratic Hamiltonians, the classification of equilibria and bifurcations in different regions of the parametric space are well studied $[24,25,27]$.

A further step in the complexity of the problem, and in the approximation to a real one, is to consider the motion of a gyrostat under the attraction of a Newtonian field. For this problem, some authors have found approximated analytical solutions for particular cases [7, 32] and other authors have studied the equilibria and their stability when the gyrostat is in circular orbit $[33,34]$ or in the gravity field of a number of different rigid bodies [21,35]. In this paper we focus on the stability of permanent rotations of a heavy gyrostat with a fixed point, that is to say when the gyrostat is under a uniform gravity field. For this case, both necessary and sufficient conditions of stability have been obtained by means of different methods. In this sense, Rumiantsev [31] and Anchev [1,2] gave sufficient conditions of stability for permanent rotations by constructing appropriate Lyapunov functions. In the particular case the center of mass lies on the first principal axis and the gyrostatic moment is directed along the same axis, Kovalev [22] derived sufficient conditions, that matched those of Rumiantsev, but also applied KAM theory to study the stability when the associated quadratic form of the perturbed Hamiltonian is not sign definite, but the necessary conditions are satisfied.

Previous results can also be derived and improved using the Energy-Casimir method $[3,18,29,30]$ provided the system can be regarded as a Lie-Poison one. Indeed, this method has been successfully used to study rigid body dynamics [6] and recently applied to study the stability of permanent rotations of a heavy gyrostat [14]. In this paper, the authors obtain, for a special class of permanent rotations, the same results previously derived by classical methods by Kovalev [23]. For the other permanent rotations, they provide sufficient stability conditions. However, these conditions are weak, as they do not depend on the gyrostatic moment. In this paper we obtain new sufficient conditions for all the permanent rotations in the case studied in [14] and also prove that, in some configurations of the moments of inertia, they are also necessary conditions. Besides, on a certain parametric plane, we determine regions for the existence of the equilibria, as well as bifurcation lines, since the stability depends on those parameters.

\section{Equations of motion}

Let us consider a gyrostat, consisting of a rigid asymmetric platform and three axisymmetric rotors. Each one of these rotors is aligned along one of the principal axis of the platform. The gyrostat is subject to a uniform and constant 
gravity field. We assume that the gyrostat has a fixed point $O$. Centered on this point, we consider two orthonormal reference frames (see Figure 1):

- The inertial fixed reference frame $\mathcal{F}\{O, X, Y, Z\}$. The direction of the $Z$ axis is opposite to the action line of the gravity field.

- The body frame $\mathcal{B}\{O, x, y, z\}$ fixed in the platform. The directions of these axes coincide with the principal axes of the gyrostat.

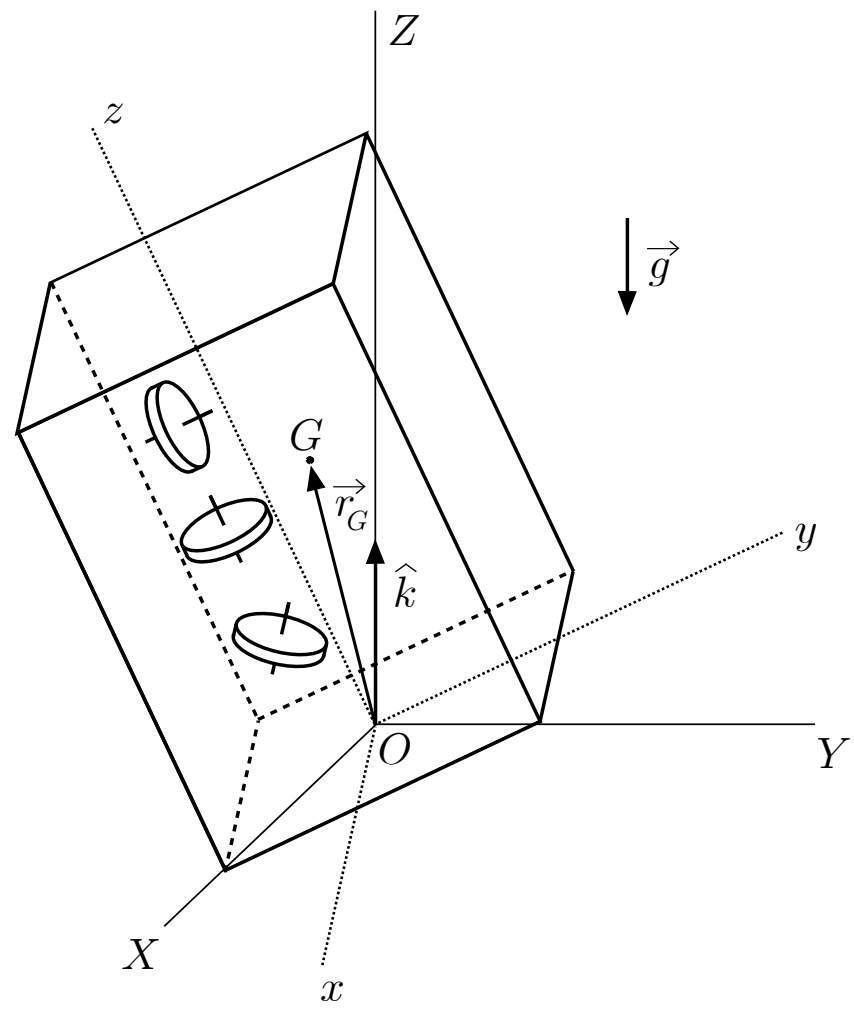

Figure 1: Asymmetric gyrostat and reference frames.

In the body reference frame $\mathcal{B}$, the tensor of inertia $\mathbb{I}$ of the gyrostat is diagonal, that is, $\mathbb{I}=\operatorname{diag}\left(I_{1}, I_{2}, I_{3}\right)$. As we assume an asymmetric gyrostat, $I_{1} \neq I_{2} \neq I_{3}$. On the other hand, the total angular momentum of the gyrostat can be written as

$$
\boldsymbol{H}=\boldsymbol{\pi}+\boldsymbol{l},
$$

where $\boldsymbol{\pi}$ is the angular momentum of the whole gyrostat with rotors at relative rest (in other words, considering the gyrostat as a rigid body), and $\boldsymbol{l}$ is the gyrostatic momentum, that is, the relative angular momentum of the rotors with respect to the platform. Due to the gravity field, the gyrostat is under the 
action of a gravitational torque $\boldsymbol{N}$ about the fixed point $O$, given by

$$
\boldsymbol{N}=\boldsymbol{r}_{G} \times m \boldsymbol{g}=-m g \boldsymbol{r}_{G} \times \hat{\boldsymbol{k}},
$$

where $\boldsymbol{r}_{G}$ is the position vector of the center of mass $G$ of the gyrostat, $\hat{\boldsymbol{k}}$ is a unitary vector in the direction of the $Z$ axis, and $m$ is the mass of the gyrostat. Under all these assumptions, and by means of the angular momemtum theorem about the fixed point $O$,

$$
\frac{d \boldsymbol{H}}{d t}=\boldsymbol{N}
$$

the Euler equations of motion expressed in the body reference frame $\mathcal{B}$ take the form $[28]$

$$
\begin{aligned}
& \frac{d \pi_{1}}{d t}=\left(\frac{I_{2}-I_{3}}{I_{2} I_{3}}\right) \pi_{2} \pi_{3}+\frac{l_{2} \pi_{3}}{I_{3}}-\frac{l_{3} \pi_{2}}{I_{2}}+m g\left(z_{0} k_{2}-y_{0} k_{3}\right), \\
& \frac{d \pi_{2}}{d t}=\left(\frac{I_{3}-I_{1}}{I_{1} I_{3}}\right) \pi_{1} \pi_{3}+\frac{l_{3} \pi_{1}}{I_{1}}-\frac{l_{1} \pi_{3}}{I_{3}}+m g\left(x_{0} k_{3}-z_{0} k_{1}\right), \\
& \frac{d \pi_{3}}{d t}=\left(\frac{I_{1}-I_{2}}{I_{1} I_{2}}\right) \pi_{1} \pi_{2}+\frac{l_{1} \pi_{2}}{I_{2}}-\frac{l_{2} \pi_{1}}{I_{1}}+m g\left(y_{0} k_{1}-x_{0} k_{2}\right),
\end{aligned}
$$

where $\left(\pi_{1}, \pi_{2}, \pi_{3}\right),\left(l_{1}, l_{2}, l_{3}\right)$ and $\left(k_{1}, k_{2}, k_{3}\right)$ are the components of the angular momenta vectors $\boldsymbol{\pi}, \boldsymbol{l}$ and the unitary vector $\hat{\boldsymbol{k}}$ respectively, expressed in the body reference frame $\mathcal{B}$. In addition, $\left(x_{0}, y_{0}, z_{0}\right)$ are the coordinates of the mass center $G$ in the same frame.

On the other hand, the components $\left(k_{1}, k_{2}, k_{3}\right)$ also vary in time as they are expressed in the body reference frame $\mathcal{B}$. The time evolution of these components is given by the well known Poisson equations [28]

$$
\begin{aligned}
& \frac{d k_{1}}{d t}=\frac{k_{2} \pi_{3}}{I_{3}}-\frac{k_{3} \pi_{2}}{I_{2}}, \\
& \frac{d k_{2}}{d t}=\frac{k_{3} \pi_{1}}{I_{1}}-\frac{k_{1} \pi_{3}}{I_{3}}, \\
& \frac{d k_{3}}{d t}=\frac{k_{1} \pi_{2}}{I_{2}}-\frac{k_{2} \pi_{1}}{I_{1}} .
\end{aligned}
$$

Therefore, equations (1) and (2) are the complete set of equations that rule the rotational dynamics of the asymmetric gyrostat under a uniform and constant gravity field. Although the variables considered are not canonical, the system can be described by means of a Hamiltonian function in the framework of LiePoisson systems, in the same way as the classical problem of the motion of a rigid body $[29,6]$. In this case, the associated Hamiltonian function takes the form (see [14])

$$
\mathcal{H}=\frac{1}{2}\left(\frac{\pi_{1}^{2}}{I_{1}}+\frac{\pi_{2}^{2}}{I_{2}}+\frac{\pi_{3}^{2}}{I_{3}}\right)+m g\left(x_{0} k_{1}+y_{0} k_{2}+z_{0} k_{3}\right),
$$


and the corresponding Poisson bracket is given by

$$
\{F, G\}(\boldsymbol{\pi}, \hat{\boldsymbol{k}})=-(\boldsymbol{\pi}+\boldsymbol{l}) \cdot\left(\nabla_{\pi} F \times \nabla_{\pi} G\right)-\hat{\boldsymbol{k}} \cdot\left(\nabla_{\pi} F \times \nabla_{k} G+\nabla_{k} F \times \nabla_{\pi} G\right) .
$$

Now, it is easy to check that the equations (1-2) of the gyrostat rotational motion can be expressed as

$$
\dot{\pi}_{i}=\left\{\pi_{i}, \mathcal{H}\right\}, \quad \dot{k_{i}}=\left\{k_{i}, \mathcal{H}\right\}, \quad i=1,2,3 .
$$

Thus, the system is regarded as a Lie-Poisson system and to study the stability of relative equilibria we can make use of the Energy-Casimir method. To this end, the existence of Casimir functions and conserved quantities plays an important role. For this problem, there are two Casimir functions whose Poisson bracket commutes with any smooth function defined in the phase space. These two Casimir functions are

$$
\begin{gathered}
C_{1} \equiv k_{1}^{2}+k_{2}^{2}+k_{3}^{2}=1 \\
C_{2} \equiv\left(\pi_{1}+l_{1}\right) k_{1}+\left(\pi_{2}+l_{2}\right) k_{2}+\left(\pi_{3}+l_{3}\right) k_{3}=p_{\psi}
\end{gathered}
$$

being $p_{\psi}$ a constant.

In what follows we will focus on a special situation, when the center of mass is located on the $z$ axis and only the gyrostatic moment along the $z$ axis is acting. Thus, $x_{0}=y_{0}=0, l_{1}=l_{2}=0$ and the equations (1-2) reduce to

$$
\begin{aligned}
\frac{d \pi_{1}}{d t} & =\frac{I_{2}-I_{3}}{I_{2} I_{3}} \pi_{2} \pi_{3}-\frac{l_{3} \pi_{2}}{I_{2}}+m g z_{0} k_{2}, \\
\frac{d \pi_{2}}{d t} & =\frac{I_{3}-I_{1}}{I_{1} I_{3}} \pi_{1} \pi_{3}+\frac{l_{3} \pi_{1}}{I_{1}}-m g z_{0} k_{1}, \\
\frac{d \pi_{3}}{d t} & =\frac{I_{1}-I_{2}}{I_{1} I_{2}} \pi_{1} \pi_{2}, \\
\frac{d k_{1}}{d t} & =\frac{k_{2} \pi_{3}}{I_{3}}-\frac{k_{3} \pi_{2}}{I_{2}}, \\
\frac{d k_{2}}{d t} & =\frac{k_{3} \pi_{1}}{I_{1}}-\frac{k_{1} \pi_{3}}{I_{3}}, \\
\frac{d k_{3}}{d t} & =\frac{k_{1} \pi_{2}}{I_{2}}-\frac{k_{2} \pi_{1}}{I_{1}} .
\end{aligned}
$$

\section{Equilibrium solutions}

Permanent rotations $[9,11,28]$ are of great interest in different fields of application and they are obtained as equilibrium solutions of the system (7). These solutions have been obtained previously by other authors [14]. However, for the sake of completeness, we give the following result. 
Theorem 1. If $x_{0}=y_{0}=0, l_{3} \neq 0$ and $l_{1}=l_{2}=0$ there are three families of equilibrium points.

$$
\begin{aligned}
E_{1} \equiv & \left(0,0, I_{3} \omega, 0,0, \pm 1\right), \quad \omega \in \mathbb{R} . \\
E_{2} \equiv & \left(0, I_{2} \omega \sin \varphi, I_{3} \omega \cos \varphi, 0, \sin \varphi, \cos \varphi\right), \\
& \text { with } \varphi \in(0,2 \pi), \quad \omega \in \mathbb{R}, \quad \text { and } \quad \omega^{2}\left(I_{3}-I_{2}\right) \cos \varphi+\omega l_{3}-g m z_{0}=0 . \\
E_{3} \equiv & \left(I_{1} \omega \sin \varphi, 0, I_{3} \omega \cos \varphi, \sin \varphi, 0, \cos \varphi\right), \\
& \text { with } \varphi \in(0,2 \pi), \quad \omega \in \mathbb{R}, \quad \text { and } \quad \omega^{2}\left(I_{3}-I_{1}\right) \cos \varphi+\omega l_{3}-g m z_{0}=0 .
\end{aligned}
$$

\section{Proof.}

Equilibria are obtained setting to zero the equations of the motion (7). Thus, it follows from the third equation of the motion that the product $\pi_{1} \pi_{2}$ must be zero.

In the first place, we consider that both $\pi_{1}$ and $\pi_{2}$ are zero. Thus, the nontrivial equations of system (7) turn to be

$$
\frac{d \pi_{1}}{d t}=m g z_{0} k_{2}, \quad \frac{d \pi_{2}}{d t}=-m g z_{0} k_{1}, \quad \frac{d k_{1}}{d t}=\frac{k_{2} \pi_{3}}{I_{3}}, \quad \frac{d k_{2}}{d t}=-\frac{k_{1} \pi_{3}}{I_{3}} .
$$

These equations vanish if $k_{1}=k_{2}=0$ and $\pi_{3}$ is any real number. By virtue of (5), we obtain two one-parameter families of equilibrium solutions, we name $E_{1}$,

$$
E_{1} \equiv\left(0,0, I_{3} \omega, 0,0, \pm 1\right)
$$

with $\omega \in \mathbb{R}$.

Now, be $\pi_{1}=0$ and $\pi_{2} \neq 0$. Then, the second and the last two equations of the motion are simultaneously equal to zero if $k_{1}=0$. Taking into account (5), we introduce an angle $\varphi \in(0,2 \pi)$ in such a way that

$$
k_{2}=\sin \varphi, \quad k_{3}=\cos \varphi .
$$

Now, the first and the fourth equations of the motion result to be

$$
\frac{d \pi_{1}}{d t}=\frac{I_{2}-I_{3}}{I_{2} I_{3}} \pi_{2} \pi_{3}-\frac{l_{3} \pi_{2}}{I_{2}}+m g z_{0} \sin \varphi, \quad \frac{d k_{1}}{d t}=\frac{\pi_{3}}{I_{3}} \sin \varphi-\frac{\pi_{2}}{I_{2}} \cos \varphi .
$$

These two equations are equal to zero if

$$
\pi_{2}=I_{2} \omega \sin \varphi, \quad \pi_{3}=I_{3} \omega \cos \varphi
$$

and $\omega$ is a real number satisfying the equation

$$
\omega^{2}\left(I_{3}-I_{2}\right) \cos \varphi+\omega l_{3}-g m z_{0}=0 .
$$

In this way, we obtain the biparametric family of equilibrium solutions named as $E_{2}$.

A similar analysis, for the case $\pi_{1} \neq 0$ and $\pi_{2}=0$, yields the third family of equilibrium solutions dubbed $E_{3}$. 


\section{Stability analysis}

In this section we will focus on the stability analysis of the equilibrium solutions given in Theorem 1 . The stability for the family $E_{1}$ has been considered in $[36,37]$ for a symmetric gyrostat. The other two families have been also considered in [14], but the stability conditions given are weak, as they do not depend on the gyrostatic moment.

Taking into account that we are considering a Poisson system, to establish sufficient stability conditions we can use the classical energy-Casimir method $[3,18]$ or a generalized version given in [30], which reads

Theorem 2 (Generalized energy-Casimir method). Let $(M,\{.,\}, h$.$) be a$ Poisson system, and $m \in M$ be an equilibrium of the Hamiltonian vector field $X_{h}$. If there is a set of conserved quantities $C_{1}, \ldots, C_{n} \in C^{\infty}(M)$ for which

$$
\mathbf{d}\left(h+C_{1}+\cdots+C_{n}\right)(m)=0,
$$

and

$$
\left.\mathbf{d}^{2}\left(h+C_{1}+\cdots+C_{n}\right)(m)\right|_{W \times W},
$$

is definite for $W$ defined by

$$
W=\operatorname{ker} \mathbf{d} C_{1}(m) \cap \cdots \cap \operatorname{ker} \mathbf{d} C_{n}(m),
$$

then $m$ is stable. If $W=\{0\}, m$ is always stable.

To begin with, we state the first stability result, concerning the equilibrium point $E_{1}$.

Theorem 3. The equilibrium $E_{1}$ is stable if the following conditions are satisfied

$$
\begin{aligned}
& \left(I_{3}-I_{1}\right) \omega^{2}+l_{3} \omega>g m z_{0}, \quad\left(I_{3}-I_{2}\right) \omega^{2}+l_{3} \omega>g m z_{0}, \quad k_{3}=1 . \\
& \left(I_{3}-I_{1}\right) \omega^{2}+l_{3} \omega>-g m z_{0}, \quad\left(I_{3}-I_{2}\right) \omega^{2}+l_{3} \omega>-g m z_{0}, \quad k_{3}=-1 .
\end{aligned}
$$

Proof. The proof can be found in [14], Theorem 7 .

This result can be complemented by the necessary conditions of stability, also given in [23]. In this sense, we have the following Theorem.

Theorem 4. If the equilibrium $E_{1}$ is stable, then it must be satisfied

$$
\begin{array}{ll}
{\left[\left(I_{3}-I_{1}\right) \omega^{2}+l_{3} \omega-g m z_{0}\right]\left[\left(I_{3}-I_{2}\right) \omega^{2}+l_{3} \omega-g m z_{0}\right]>0,} & k_{3}=1 . \\
{\left[\left(I_{3}-I_{1}\right) \omega^{2}+l_{3} \omega+g m z_{0}\right]\left[\left(I_{3}-I_{2}\right) \omega^{2}+l_{3} \omega+g m z_{0}\right]>0,} & k_{3}=-1 .
\end{array}
$$

Proof. The proof is straightforward, taking into account that a necessary condition to be stable is to be linearly stable. Thus, none of the eigenvalues 
of the linearized system can have positive real part. However, the linearized system around $E_{1}$, for the case $k_{3}=1$, is defined by the Jacobian matrix

$$
J_{E_{1}}=\left(\begin{array}{cccccc}
0 & \frac{\left(I_{2}-I_{3}\right) \omega-l_{3}}{I_{2}} & 0 & 0 & g m z_{0} & 0 \\
\frac{\left(I_{3}-I_{1}\right) \omega+l_{3}}{I_{1}} & 0 & 0 & -g m z_{0} & 0 & 0 \\
0 & 0 & 0 & 0 & 0 & 0 \\
0 & -\frac{1}{I_{2}} & 0 & 0 & \omega & 0 \\
\frac{1}{I_{1}} & 0 & 0 & -\omega & 0 & 0 \\
0 & 0 & 0 & 0 & 0 & 0
\end{array}\right) .
$$

The eigenvalues of $J_{E_{1}}$ are the roots of the characteristic polynomial, which has the following form

$$
\lambda^{2}\left(\lambda^{4}+a \lambda^{2}+b\right) .
$$

It is clear that there are two eigenvalues equal to 0 and, for the remaining four eigenvalues, it follows that if $\lambda_{0}$ is an eigenvalue, also $-\lambda_{0}, \bar{\lambda}_{0}$ and $-\bar{\lambda}_{0}$ are eigenvalues. Thus, a necessary condition for $E_{1}$ to be linear stable is that eigenvalues have zero real part, which means that the coefficient $b$ must be greater than 0 . However,

$$
b=\frac{1}{I_{1} I_{2}}\left[\left(I_{3}-I_{1}\right) \omega^{2}+l_{3} \omega-g m z_{0}\right]\left[\left(I_{3}-I_{2}\right) \omega^{2}+l_{3} \omega-g m z_{0}\right],
$$

and the first case is proved. The case $k_{3}=-1$ can be proved in the same way.

Note that, in the case the two expressions in brackets are positive, necessary and sufficient conditions are exactly the same.

Remark 1. It is worth to mention that in the axisymmetric case, $I_{2}=I_{1}$, the two stability conditions, for $k_{3}=1$ (similarly for $k_{3}=-1$ ), reduce to one

$$
\left(I_{3}-I_{1}\right) \omega^{2}+l_{3} \omega>g m z_{0} .
$$

However, this is a different stability condition of the classical one [36]

$$
\left(I_{3} \omega+l_{3}\right)^{2} \geq 4 g m z_{0} I_{1} .
$$

This is due to the fact that, in the augmented Hamiltonian used to prove the stability in Theorem 3, the conservation of the third component of the angular momentum, appearing in the symmetric case, cannot be considered. Thus, the stability condition is more restrictive, in the sense that if (8) is satisfied, also (9) is satisfied, but not necessarily in the reverse way. Indeed,

$$
\frac{\left(I_{3} \omega+l_{3}\right)^{2}}{4 I_{1}} \geq\left(I_{3}-I_{1}\right) \omega^{2}+l_{3} \omega
$$



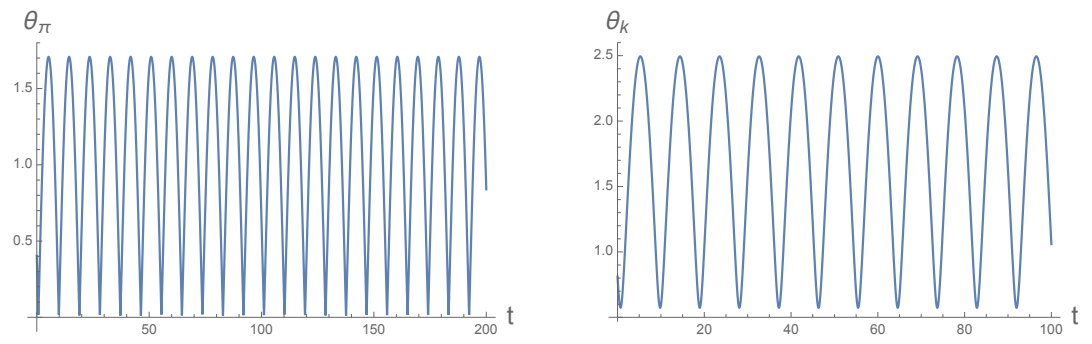

Figure 2: The angle formed by the vectors $\left(\pi_{1}, \pi_{2}, \pi_{3}\right)$ and $\left(k_{1}, k_{2}, k_{3}\right)$ with the corresponding equilibrium position $E_{1},(0,0,2),(0,0,1)$, for the case $I_{1}=I_{2}=1, I_{3}=2, m g z_{0}=2, \omega=1$ and $l_{3}=0.9$. The initial conditions of the trajectory are $(0.01,0.01,2.01,0.0141418,0,0.9999)$ and the angle is measured in degrees.

provided that

$$
\left(I_{3} \omega+l_{3}\right)^{2}-4 I_{1}\left(\left(I_{3}-I_{1}\right) \omega^{2}+l_{3} \omega\right)=\left(I_{3} \omega+l_{3}-2 I_{1} \omega\right)^{2} \geq 0 .
$$

To emphasize this situation, we consider a gyrostat with the following values for the parameters

$$
I_{1}=I_{2}=1, \quad I_{3}=2, \quad g m z_{0}=2, \quad \omega=1, \quad l_{3}=0.9 .
$$

It is easy to check that

$$
\left(I_{3}-I_{1}\right) \omega^{2}+l_{3} \omega=1.9<g m z_{0}=2
$$

and

$$
\left(I_{3} \omega+l_{3}\right)^{2}=8.41>4 m g z_{0} I_{1}=8 .
$$

That is to say, stability condition (9) is satisfied, but not condition (8). Numerical integration of a trajectory starting close to the equilibrium position $(0,0,2,0,0,1)$ shows that it remains close to the equilibrium point along time. As we move away from the equilibrium, the trajectories stay close to it, but performing a precession movement. This can be seen in Figure 2.

Now, we state a result about the stability of the second and third families of permanent rotations, $E_{2}$ and $E_{3}$, which turns to be more general than that one given in [14].

Theorem 5. The equilibrium $E_{2}$ is stable if the following conditions are satisfied

$$
I_{1}>I_{3}, \quad l_{2}^{2}>\left(I_{2}-I_{1}\right)\left(I_{1} w^{2}-4 l_{3} \omega \cos \varphi+3\left(I_{1}-I_{2}\right) \omega^{2} \cos ^{2} \varphi\right) .
$$

In particular, if $\cos \varphi=0, E_{2}$ is stable if

$$
I_{1}>I_{3}, \quad l_{3}^{4}>I_{1}\left(I_{2}-I_{1}\right) m^{2} g^{2} z_{0}^{2} .
$$


Analogously, the equilibrium $E_{3}$ is stable if the following conditions are satisfied

$$
I_{1}>I_{2}, \quad l_{3}^{2}>\left(I_{3}-I_{1}\right)\left(I_{1} w^{2}-4 l_{3} \omega \cos \varphi+3\left(I_{1}-I_{3}\right) \omega^{2} \cos ^{2} \varphi\right) .
$$

In particular, if $\cos \varphi=0, E_{3}$ is stable if

$$
I_{1}>I_{2}, \quad l_{3}^{4}>I_{1}\left(I_{3}-I_{1}\right) m^{2} g^{2} z_{0}^{2} .
$$

Proof. We will perform the proof for the equilibrium $E_{3}$, as the other case is exactly the same, interchanging the role played by the moments of inertia $I_{2}$ and $I_{3}$. Following Theorem 2, we introduce the augmented Hamiltonian

$H=\frac{1}{2}\left(\frac{\pi_{1}^{2}}{I_{1}}+\frac{\pi_{2}^{2}}{I_{2}}+\frac{\pi_{3}^{2}}{I_{3}}\right)+m g z_{0} k_{3}+\lambda\left(\pi_{1} k_{1}+\pi_{2} k_{2}+\left(l_{3}+\pi_{3}\right) k_{3}\right)+\mu\left(k_{1}^{2}+k_{2}^{2}+k_{3}^{2}\right)$,

where to the Hamiltonian function (3) we have added a linear combination of the two Casimir functions (5) and (6). It is easy to check that equilibrium $E_{3}$ is a critical point of $H$ if the parameters $\lambda$ and $\mu$ are given by

$$
\lambda=-\omega, \quad \mu=\frac{I_{1} \omega^{2}}{2} .
$$

Let us now determine the space

$$
W=\operatorname{ker} \mathbf{d} C_{1}\left(E_{3}\right) \cap \operatorname{ker} \mathbf{d} C_{2}\left(E_{3}\right),
$$

where $C_{1}$ and $C_{2}$ are the Casimir functions already given by (5) and (6) and introduced in the augmented Hamiltonian (10). On the one hand, we have

$$
\mathbf{d} C_{1}\left(E_{3}\right)=2 \sin \varphi d k_{1}+2 \cos \varphi d k_{3}
$$

and, on the other hand,

$$
\mathbf{d} C_{2}\left(E_{3}\right)=\sin \varphi d \pi_{1}+\cos \varphi d \pi_{3}+I_{1} \omega \sin \varphi d k_{1}+\left(l_{3}+I_{3} \omega \cos \varphi\right) d k_{3} .
$$

Equating to zero the above expressions, we obtain the relations

$$
d \pi_{3}=-d \pi_{1} \tan \varphi+\left(\frac{l_{3}}{\cos \varphi}+\left(I_{3}-I_{1}\right) \omega\right) \tan \varphi d k_{1}, \quad d k_{3}=-d k_{1} \tan \varphi .
$$

Thus, $W=\operatorname{ker} \mathbf{d} C_{1}\left(E_{3}\right) \cap \operatorname{ker} \mathbf{d} C_{2}\left(E_{3}\right)$ is generated by the vectors

$\hat{\mathbf{e}}_{1} \cos \varphi-\hat{\mathbf{e}}_{3} \sin \varphi, \hat{\mathbf{e}}_{2}, \hat{\mathbf{e}}_{3}\left[l_{3}+\left(I_{3}-I_{1}\right) \omega \cos \varphi\right] \sin \varphi+\hat{\mathbf{e}}_{4} \cos ^{2} \varphi-\hat{\mathbf{e}}_{6} \sin \varphi \cos \varphi, \hat{\mathbf{e}}_{5}$.

Let $\boldsymbol{v}$ be a six dimensional vector in $W$, then

$$
\begin{aligned}
\boldsymbol{v}= & \left(x_{1} \cos \varphi, x_{2},\right. \\
& \left.-\left(x_{1}-l_{3} x_{3}+\left(I_{1}-I_{3}\right) \omega x_{3} \cos \varphi\right) \sin \varphi, x_{3} \cos ^{2} \varphi, x_{4},-x_{3} \cos \varphi \sin \varphi\right),
\end{aligned}
$$


where $x_{i} \in \mathbb{R}, i=1, \ldots, 4$. Then, the Hessian matrix of the augmented Hamiltonian in the reduced space $W$ is computed straightforwardly from the quadratic form in the variables $x_{i}$,

$$
\boldsymbol{v}^{\mathrm{T}} \cdot\left[\begin{array}{cccccc}
\frac{\partial^{2} H}{\partial \pi_{1}^{2}} & \frac{\partial^{2} H}{\partial \pi_{1} \pi_{2}} & \frac{\partial^{2} H}{\partial \pi_{1} \pi_{3}} & \frac{\partial^{2} H}{\partial \pi_{1} k_{1}} & \frac{\partial^{2} H}{\partial \pi_{1} k_{2}} & \frac{\partial^{2} H}{\partial \pi_{1} k_{3}} \\
\frac{\partial^{2} H}{\partial \pi_{1} \pi_{2}} & \frac{\partial^{2} H}{\partial \pi_{2}^{2}} & \frac{\partial^{2} H}{\partial \pi_{2} \pi_{3}} & \frac{\partial^{2} H}{\partial \pi_{2} k_{1}} & \frac{\partial^{2} H}{\partial \pi_{2} k_{2}} & \frac{\partial^{2} H}{\partial \pi_{2} k_{3}} \\
\frac{\partial^{2} H}{\partial \pi_{1} \pi_{3}} & \frac{\partial^{2} H}{\partial \pi_{2} \pi_{3}} & \frac{\partial^{2} H}{\partial \pi_{3}^{2}} & \frac{\partial^{2} H}{\partial \pi_{3} k_{1}} & \frac{\partial^{2} H}{\partial \pi_{3} k_{2}} & \frac{\partial^{2} H}{\partial \pi_{3} k_{3}} \\
\frac{\partial^{2} H}{\partial \pi_{1} k_{1}} & \frac{\partial^{2} H}{\partial \pi_{2} k_{1}} & \frac{\partial^{2} H}{\partial \pi_{3} k_{1}} & \frac{\partial^{2} H}{\partial k_{1}^{2}} & \frac{\partial^{2} H}{\partial k_{1} k_{2}} & \frac{\partial^{2} H}{\partial k_{1} k_{3}} \\
\frac{\partial^{2} H}{\partial \pi_{1} k_{2}} & \frac{\partial^{2} H}{\partial \pi_{2} k_{2}} & \frac{\partial^{2} H}{\partial \pi_{3} k_{2}} & \frac{\partial^{2} H}{\partial k_{1} k_{2}} & \frac{\partial^{2} H}{\partial k_{2}^{2}} & \frac{\partial^{2} H}{\partial k_{2} k_{3}} \\
\frac{\partial^{2} H}{\partial \pi_{1} k_{3}} & \frac{\partial^{2} H}{\partial \pi_{2} k_{3}} & \frac{\partial^{2} H}{\partial \pi_{3} k_{3}} & \frac{\partial^{2} H}{\partial k_{1} k_{3}} & \frac{\partial^{2} H}{\partial k_{2} k_{3}} & \frac{\partial^{2} H}{\partial k_{3}^{2}}
\end{array}\right] \cdot \boldsymbol{v}
$$

where the full Hessian matrix is evaluated at $E_{3}$. In this way, we arrive to

$$
\left.\mathrm{Hess}\right|_{W \times W}=\left[\begin{array}{cccc}
\frac{\cos ^{2} \varphi}{I_{1}}+\frac{\sin ^{2} \varphi}{I_{3}} & 0 & H_{13} & 0 \\
0 & \frac{1}{I_{2}} & 0 & -\omega \\
H_{13} & 0 & \frac{\varkappa}{I_{3}} & 0 \\
0 & -\omega & 0 & I_{1} \omega^{2}
\end{array}\right] \text {, }
$$

where

$$
H_{13}=\frac{-I_{3} \omega \cos ^{3} \varphi-l_{3} \sin ^{2} \varphi+\left(I_{1}-2 I_{3}\right) \omega \cos \varphi \sin ^{2} \varphi}{I_{3}}
$$

and

$$
\begin{aligned}
\varkappa= & I_{1} I_{3} \omega^{2} \cos ^{4} \varphi+l_{3}^{2} \sin ^{2} \varphi-2\left(I_{1}-2 I_{3}\right) l_{3} \omega \cos \varphi \sin ^{2} \varphi \\
& +\left(I_{1}^{2}-3 I_{1} I_{3}+3 I_{3}^{2}\right) \omega^{2} \cos ^{2} \varphi \sin ^{2} \varphi .
\end{aligned}
$$

Now, we apply the Sylvester criterion to determine the definiteness of the matrix, computing the principal minors. They are given by

$$
\begin{aligned}
& \Delta_{1}=\frac{\cos ^{2} \varphi}{I_{1}}+\frac{\sin ^{2} \varphi}{I_{3}}, \quad \Delta_{2}=\frac{\Delta_{1}}{I_{2}}, \\
& \Delta_{3}=\frac{l_{3}^{2}-\left(I_{3}-I_{1}\right)\left(I_{1} w^{2}-4 l_{3} \omega \cos \varphi+3\left(I_{1}-I_{3}\right) \omega^{2} \cos ^{2} \varphi\right)}{4 I_{1} I_{2} I_{3}} \sin ^{2} 2 \varphi, \\
& \Delta_{4}=\left(I_{1}-I_{2}\right) \omega^{2} \Delta_{3} .
\end{aligned}
$$

It is clear that $\Delta_{1}$ and $\Delta_{2}$ are always positive, despite the value of the parameter $\varphi \in(0,2 \pi)$. The other two minors are positive if the following inequalities are satisfied

$$
l_{3}^{2}-\left(I_{3}-I_{1}\right)\left(I_{1} w^{2}-4 l_{3} \omega \cos \varphi+3\left(I_{1}-I_{3}\right) \omega^{2} \cos ^{2} \varphi\right)>0, \quad I_{1}>I_{2} .
$$


In the special case $\cos \varphi=0$, we can obtain $\omega$ from the relation satisfied by $\omega$ and $\varphi$ for the existence of the equilibrium $E_{3}$. That is,

$$
w^{2}\left(I_{3}-I_{1}\right) \cos \varphi+\omega l_{3}-g m z_{0}=0 .
$$

As $\cos \varphi=0$ it follows

$$
\omega=\frac{g m z_{0}}{l_{3}}
$$

and (11) reduces to

$$
l_{3}^{4}>I_{1}\left(I_{3}-I_{1}\right) m^{2} g^{2} z_{0}^{2}, \quad I_{1}>I_{2} .
$$

Remark 2. It is worth noticing that if $I_{1}>I_{3}$ the first condition in (11) is always satisfied.

Indeed, taking into account the existence relation for the family $E_{3}$

$$
\omega^{2}\left(I_{3}-I_{1}\right) \cos \varphi+\omega l_{3}-g m z_{0}=0
$$

we obtain that

$$
l_{3}=\left(I_{1}-I_{3}\right) \omega+\frac{g m z_{0}}{\omega} .
$$

Substituting this relation in (11) we arrive to the equivalent inequality

$$
\left.I_{1}\left(I_{1}-I_{3}\right) \omega^{4}-2 g m z_{0} I_{1}-I_{3}\right) \omega^{2} \cos \varphi+g^{2} m^{2} z_{0}^{2}>0 .
$$

However, the left hand side of the inequality is a biquadratic polynomial in $\omega$, with roots

$$
\omega^{2}=g m z_{0} \frac{\left(I_{1}-I_{3}\right) \cos \varphi \pm \sqrt{\left(I_{3}-I_{1}\right)\left(I_{1} \sin ^{2} \varphi+I_{3} \cos ^{2} \varphi\right)}}{I_{1}\left(I_{1}-I_{3}\right)} .
$$

It is clear that there are no real roots if $I_{1}>I_{3}$. Taking into account that the coefficient of the leading term is $I_{1}\left(I_{1}-I_{3}\right)>0$, the first inequality is satisfied. In this way, we obtain the weak stability conditions

$$
I_{1}>I_{2}, \quad I_{1}>I_{3} .
$$

Thus, if $I_{1}$ is the biggest moment of inertia, it does not matter the value of the gyrostatic moment $l_{3}$, the equilibrium position $E_{3}$ is always stable. This is precisely the conclusion in [14]. However, the first inequality in (11) is more general and we can obtain stability in different situations, when $I_{1}$ is not the biggest moment of inertia.

It is also remarkable that, in the case $I_{1}>I_{2}$, the sufficient condition established in Theorem 5 is also a necessary condition. Indeed, we have the following result. 
Theorem 6. A necessary condition for the equilibrium $E_{3}$ to be stable is

$$
\left(I_{1}-I_{2}\right)\left(l_{3}^{2}-\left(I_{3}-I_{1}\right)\left(I_{1} \omega^{2}-4 l_{3} \omega \cos \varphi+3\left(I_{1}-I_{3}\right) \omega^{2} \cos ^{2} \varphi\right)\right)>0 .
$$

Proof. Linear stability is necessary to have Lyapunov stability. In this way, as the system is Hamiltonian, eigenvalues of the linearized system come in quadruplets of the form $\pm a \pm b i$ and linear stability takes place if the real part of the eigenvalues are equal to zero.

The eigenvalues are the roots of the polynomial equation

$$
\operatorname{det}\left(J_{E_{3}}-\lambda I_{6}\right)=0,
$$

where $I_{6}$ is the $6 \times 6$ identity matrix and $J_{E_{3}}$ is the Jacobian matrix of the linearized system at $E_{3}$. This matrix results to be

$$
J_{E_{3}}=\left[\begin{array}{cccccc}
0 & J_{12} & 0 & 0 & m g z_{0} & 0 \\
J_{21} & 0 & J_{23} & -m g z_{0} & 0 & 0 \\
0 & J_{32} & 0 & 0 & 0 & 0 \\
0 & -\frac{\cos \varphi}{I_{2}} & 0 & 0 & \omega \cos \varphi & 0 \\
\frac{\cos \varphi}{I_{1}} & 0 & -\frac{\sin \varphi}{I_{3}} & -\omega \cos \varphi & 0 & \omega \sin \varphi \\
0 & \frac{\sin \varphi}{I_{2}} & 0 & 0 & -\omega \sin \varphi & 0
\end{array}\right],
$$

where

$$
\begin{array}{ll}
J_{12}=\frac{-l_{3}+\left(I_{2}-I_{3}\right) \omega \cos \varphi}{I_{2}}, & J_{21}=\frac{l_{3}-\left(I_{1}-I_{3}\right) \omega \cos \varphi}{I_{1}}, \\
J_{23}=-\frac{\left(I_{1}-I_{3}\right) \omega \sin \varphi}{I_{3}}, & J_{32}=\frac{\left(I_{1}-I_{2}\right) \omega \sin \varphi}{I_{2}} .
\end{array}
$$

The polynomial equation (13) turns to be of the form

$$
\lambda^{2}\left(\lambda^{4}+b \lambda^{2}+c\right)=0,
$$

with $b$ and $c$ real numbers. It is clear that if all the roots of equation (14) have zero real part, then $c>0$. However,

$$
c=\frac{\omega^{2} \sin ^{2} \varphi}{I_{1} I_{2} I_{3}}\left(I_{1}-I_{2}\right)\left[l_{3}^{2}-\left(I_{3}-I_{1}\right)\left(I_{1} \omega^{2}-4 l_{3} \omega \cos \varphi+3\left(I_{1}-I_{3}\right) \omega^{2} \cos ^{2} \varphi\right)\right],
$$

and the result follows immediately, by taking into account that the moments of inertia are always positive.

Theorems 5 and 6 give us a complete characterization of the stability properties of $E_{3}$ if $I_{1}>I_{2}$. Now, we are in position to obtain a picture of the 
stability regions in terms of the relevant parameters of the problem: $\varphi$ and $l_{3}$, the gyrostatic moment. The rest of the parameters stands for the geometry of the gyrostat and the position of the center of mass. For a prescribed geometry, the equilibrium $E_{3}$ exists if $\omega$ is a real number. Solving (12) to obtain $\omega$, we find that $E_{3}$ exists if

$$
l_{3}^{2}-4 g m z_{0}\left(I_{1}-I_{3}\right) \cos \varphi \geq 0 .
$$

When the inequality is transformed in an equality, it defines a curve in the plane $\left(\varphi, l_{3}\right)$ dividing it into two regions, one of them for the region of existence of $E_{3}$ and the other one for the region where $E_{3}$ does not exist. We note that the existence region depends on the sign of $\left(I_{1}-I_{3}\right) z_{0}$. In the same way, there is a curve that separates the stability and instability regions. To describe these regions is sufficient to study the case $I_{1}<I_{3}$ because, if $I_{1}>I_{3}$, the stability conditions are always satisfied (Remark 2) and, therefore, the stability region is the same as the existence region. For the case $I_{1}<I_{3}$, we have to proceed carefully as, once $l_{3}$ and $\varphi$ are fixed, two different values of $\omega$ are obtained

$$
\omega_{ \pm}=\frac{-l_{3} \pm \sqrt{l_{3}^{2}+4\left(I_{3}-I_{1}\right) g m z_{0} \cos \varphi}}{2\left(I_{3}-I_{1}\right) \cos \varphi}
$$

and, therefore, also two equilibrium points we name $E_{3+}$ and $E_{3-}$, corresponding to the values $\omega_{+}$and $\omega_{-}$respectively. Substituting these two expressions into the first inequality in (11) we arrive to the limiting curve

$$
\begin{aligned}
& I_{1}\left(l_{3}^{4}+g^{2} m^{2} z_{0}^{2} I_{1}\left(I_{1}-I_{3}\right)\right) \\
& \quad-6 g m z_{0} I_{1} l_{3}^{2}\left(I_{1}-I_{3}\right) \cos \varphi+6 g^{2} m^{2} z_{0}^{2} I_{1}\left(I_{1}-I_{3}\right)^{2} \cos ^{2} \varphi \\
& \quad-2 g m z_{0} l_{3}^{2}\left(I_{1}-I_{3}\right)^{2} \cos ^{3} \varphi+9 g^{2} m^{2} z_{0}^{2}\left(I_{1}-I_{3}\right)^{3} \cos ^{4} \varphi=0,
\end{aligned}
$$

that separates the stability and instability regions.

It is worth noting that Eqs. (15) and (17) do not depend on $I_{2}$, but only on $I_{1}$ and $I_{3}$. Moreover, $\left(I_{1}-I_{3}\right)$ appears as a relevant quantity. For this reason we introduce the quantities

$$
a=I_{3}-I_{1}, \quad b=\frac{I_{3}-I_{1}}{I_{1}},
$$

as the parameters to describe the geometry of the gyrostat. In terms of $a$ and $b$, the curves delimiting (15) and (17) become respectively

$$
\begin{aligned}
& f_{1}\left(l_{3}, \varphi ; a, b, g m z_{0}\right) \equiv l_{3}^{2}+4 a g m z_{0} \cos \varphi=0, \\
& f_{2}\left(l_{3}, \varphi ; a, b, g m z_{0}\right) \equiv-b l_{3}^{4}+a^{2} g^{2} m^{2} z_{0}^{2}-6 a b g m z_{0} l_{3}^{2} \cos \varphi \\
& -6 a^{2} b g^{2} m^{2} z_{0}^{2} \cos ^{2} \varphi+2 a b^{2} g m z_{0} l_{3}^{2} \cos ^{3} \varphi+9 a^{2} b^{2} g^{2} m^{2} z_{0}^{2} \cos ^{4} \varphi=0 .
\end{aligned}
$$

The two curves are double symmetric since for $i=1,2$,

$$
\begin{aligned}
& f_{i}\left(l_{3}, \varphi ; a, b, g m z_{0}\right)=f_{i}\left(-l_{3}, \varphi ; a, b, g m z_{0}\right), \\
& f_{i}\left(l_{3}, \pi+\varphi ; a, b, g m z_{0}\right)=f_{i}\left(l_{3}, \pi-\varphi ; a, b, g m z_{0}\right) .
\end{aligned}
$$



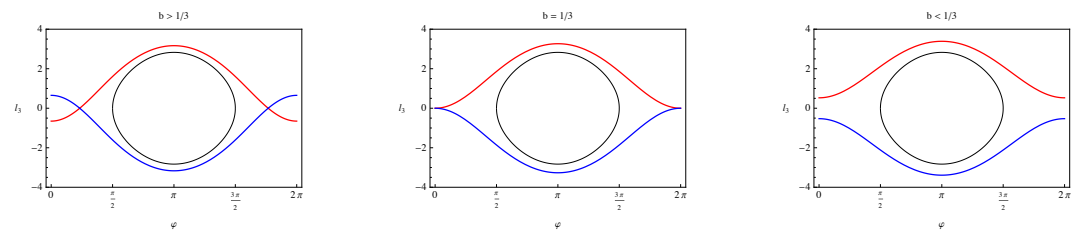

Figure 3: The two branches of the curve defined $f_{2}=0$ for different values of $b$ and $z_{0}>0$. The red branch comes from $w_{+}$, and the blue one from $\omega_{-}$. The area inside the black curve corresponds to the region where the family of equilibrium points $E_{3}$ does not exist.

We also note that $a b>0$ and that, for $a<0$ and $b<0$, the curve defined by $f_{2}$ does not exist because, as it was proven in Remark 2 , when $I_{1}>I_{3}$ equilibrium $E_{3}$ is stable and, consequently, $f_{2} \neq 0$.

We stress that, in fact, $f_{2}$ is a two branched curve, one of its branches coming from $\omega_{+}$and the other one from $\omega_{-}$and these branches are different depending on the sign of $z_{0}$. For the case $z_{0}>0$, the two branches intersect each other at the points

$$
\left(\varphi=\arccos \frac{1}{\sqrt{3 b}}, l_{3}=0\right), \quad\left(\varphi=2 \pi-\arccos \frac{1}{\sqrt{3 b}}, l_{3}=0\right),
$$

provided $b \geq 1 / 3$. For $b<1 / 3$ the two branches do not cross. The aspect of the branches for different values of $b$ is depicted in Figure 3. The red branch stands for the value $\omega_{+}$and the blue one for the value of $\omega_{-}$. When the red branch is crossed the corresponding equilibrium point $E_{3+}$ changes its stability character. In this way, it is easy to check that $E_{3+}$ is stable above the red branch and unstable below it. For $E_{3-}$ the situation is the opposite, the equilibrium point is stable below the blue branch and unstable above it.

The previous considerations show that for each equilibrium point of the family $E_{3}$ there is a critical value of the gyrostatic moment in such a way that if it is crossed, the stability changes. Some members of the family $E_{3+}$ require positive values of the gyrostatic moment to be stable and the other ones, $E_{3-}$, negative values. Nevertheless, the geometry of the gyrostat is also important. Indeed, if $b>1 / 3$, small absolute values of $l_{3}$ give rise to stable points if the angle $\varphi$ verifies $\cos \varphi>1 / \sqrt{3 b}$. On the other hand, if $b>1 / 3$ and

$$
l_{3}^{2}<a g m z_{0}\left(-3+b+\frac{(1+b)^{3 / 2}}{\sqrt{b}}\right),
$$

every member of the family $E_{3}$ is unstable, regardless the value of the angle $\varphi$. Figure 4 summarizes this. There, the stability regions for the two equilibrium points associated to a pair $\left(\varphi, l_{3}\right)$ are shown. The light green area indicates stability for the equilibrium $E_{3+}$ and instability for the other. The dark green area stands for the stable region for $E_{3-}$ and instability for the other. The red zone indicates instability for both equilibrium points and the blue one stability for the two. 

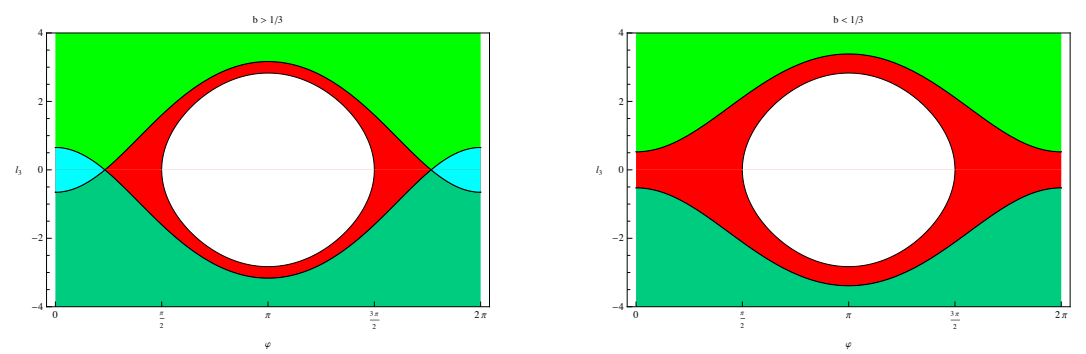

Figure 4: Stability regions for the two equilibrium points associated to a pair $\left(\varphi, l_{3}\right)$. The green areas (light and dark) indicates stability for one of the equilibrium points and instability for the other. Red and blue zones stand for unstable and stable regions for both equilibria, respectively.
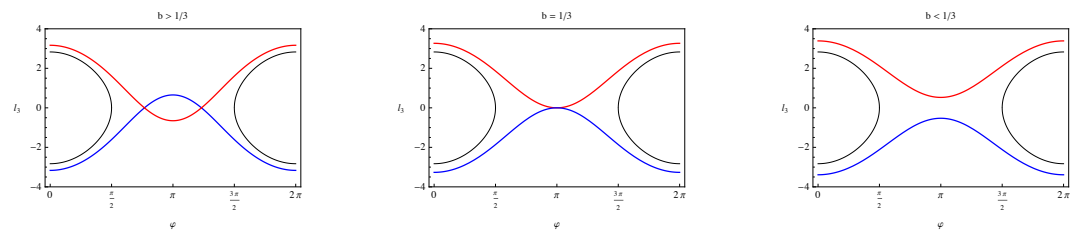

Figure 5: The two branches of the curve defined $f_{2}=0$ for different values of $b$ and $z_{0}<0$. The red branch comes from $w_{+}$, and the blue one from $\omega_{-}$. The area inside the black curve corresponds to the region where the family of equilibrium points $E_{3}$ does not exist.

A similar analysis can be made for $z_{0}<0$ obtaining similar results. Indeed, we obtain exactly the same but interchanging the intervals $[0, \pi / 2] \cup[3 \pi / 2,2 \pi]$ and $[\pi / 2,3 \pi / 2]$. Now the branches associated to the two values $\omega_{ \pm}$intersect at the points

$$
\left(\varphi=\arccos \frac{-1}{\sqrt{3 b}}, l_{3}=0\right), \quad\left(\varphi=2 \pi-\arccos \frac{-1}{\sqrt{3 b}}, l_{3}=0\right),
$$

provided $b \geq 1 / 3$, otherwise they do not intersect. Figure 5 shows the two branches for different values of $b$. As in the case $z_{0}>0$, when the red branch is crossed from above to below the equilibrium point $E_{3+}$ changes its character from stable to unstable. When the blue branch is crossed from above to below, the equilibrium point $E_{3-}$ changes from unstable to stable.

A different approach to the stability regions can be made if we consider as the relevant parameters $\varphi$ and $\omega$. Now, fixed a pair $(\varphi, \omega), \omega \neq 0$, there is only one $l_{3}$ defining an equilibrium point. Thus, we do not have to face the analysis of the branches and also we do not have to take care about the region where the equilibrium exists. In this way, proceeding as above, we obtain $l_{3}$ from (12), then introduce its expression into the first inequality in (11) and we obtain that the stability area is delimited by the curves defined by $f_{3}=0$, where $f_{3}$ is the 

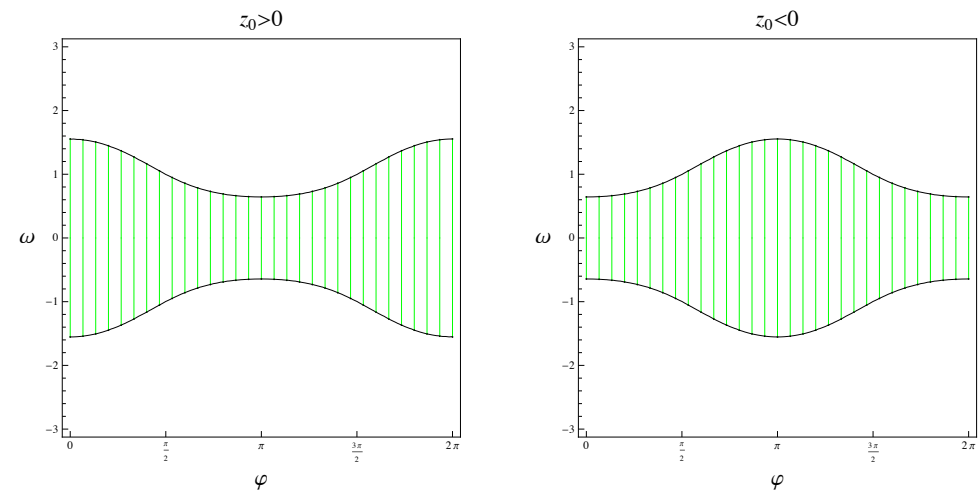

Figure 6: The two branches of the curve defined by $f_{3}=0$ for $z_{0}>0$ and $z_{0}<0, a=b=1$. Stability takes place in between them.

function

$$
f_{3} \equiv-a^{2} \omega^{4}+b g^{2} m^{2} z_{0}^{2}+2 a b g m \omega^{2} z_{0} \cos \varphi .
$$

This is a two branched curve, and the branches are different depending on the sign of $z_{0}$. In Figure 6 the two cases are depicted and stability takes place in the bounded region between the two branches. To account for the effect of $l_{3}$, we can fix its value and depict the corresponding line defined in the $(\varphi, \omega)$ plane by the existence condition (12). Different curves are shown in Figure 7, where it can be seen what pairs of $(\varphi, \omega)$ are stable for a prescribed value of $l_{3}$. It is worth noticing the pseudo symmetry for the positive and negative values of $l_{3}$. Indeed, the curves for $l_{3}>0$ and $l_{3}<0$ are symmetric with respect the axis $\omega=0$. That means that if a pair $\left(\varphi_{0}, \omega_{0}\right)$ is stabilized by $l_{3}$, the pair $\left(\varphi_{0},-\omega_{0}\right)$ is stabilized by $-l_{3}$. Furthermore, it can also be observed the behavior described previously in Figure 4, that is, how for small values of $\left|l_{3}\right|$ and small $\varphi_{0}$ we can find two values of $\omega$ for which the corresponding equilibrium is stable.

Remark 3. It is interesting to note that if $I_{2}>I_{1}$ the above described stability regions seem to change. That is to say, stability turns to be instability and vice versa. However, a detailed study, using KAM theory must be performed, as some instabilities can appear due to the presence of resonances.

\section{Conclusions}

The stability of the permanent rotations of a heavy gyrostat with a fixed point, whose center of mass is located in one of the principal axis and the gyrostatic moment acts along the same axis, has been studied by means of the Energy-Casimir method. First of all, we have established the existence of three families of permanent rotations. For the first family, we have obtained sufficient and necessary stability conditions, and we have proved that they are the same in 


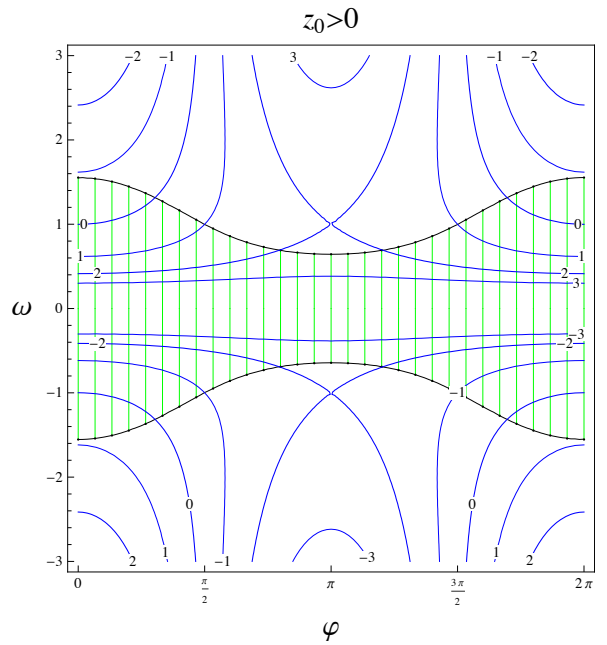

Figure 7: Different curves for the gyrostatic moment onto the stability plane in $(\varphi, \omega)$ for $a=b=1$ and $z_{0}>0$. The part of the curve inside the shaded region gives rise to a stable equilibrium point, whereas the equilibrium is unstable in the outer part.

half the region where the necessary conditions are satisfied. Moreover, we have stressed that these conditions does not approach, in the limit, to the classical conditions for a axisymmetric gyrostat, due to the appearance of a conserved quantity for the symmetric case. The other two families can be treated at once, as they are, in some sense, symmetric. For these two families the vector defining the permanent rotation lies in the plane of two of the principal axes and the third one is orthogonal to it. For these families it is proved that the stability conditions are independent of the value of the moment of inertia corresponding to the orthogonal principal axis. Besides, if the other two moments of inertia verify that the one corresponding to the principal axis where the center of mass lies is not the biggest, then the permanent rotations are always stable, does not matter the value of the gyrostatic moment. On the other hand, if it is the biggest, then a minimum gyrostatic moment is necessary to stabilize the rotations. This fact is analyzed in detail in a suitable parametric plane.

\section{Acknowledgements}

This work has been partially supported by the Spanish Ministry of Economy, projects MTM2011-28227-C02-02, MTM2014-59433-C2-2-P and ESP201344217-R. A.E. also acknowledges support from the group E-48 of the Aragon Government and FEDER funds. 


\section{References}

[1] A. Anchev, On the stability of permanent rotations of a heavy gyrostat, Pmm-J. Appl. Math. Mech. 26 (1962) 22-28.

[2] A. Anchev, Permanent rotations of a heavy gyrostat having a stationary point, Pmm-J. Appl. Math. Mech. 31 (1967) 49-58.

[3] V.I. Arnold, On an a priori estimate in the theory of hydrodynamical stability, (Russian) Izv. Vyssh. Uchebn. Zaved. Mat. Nauk. 54 (1966) 3-5; (English) Am. Math. Soc. Transl. 79 (1969) 267-269.

[4] V.S. Aslanov, Integrable cases of the problem of the free motion of a gyrostat, Pmm-J. Appl. Math. Mech. 78 (2014) 445-453.

[5] V.S. Aslanov, V.V. Yudintsev, Dynamics and chaos control of asymmetric gyrostat satellites, Cosmic Res. 52 (2014) 216-228.

[6] A.M. Bloch, J.E. Marsden, Stabilization of rigid body dynamics by the Energy-Casimir method, Syst. Control Lett. 14 (1990) 341-346.

[7] J.A. Cavas, A. Vigueras, An integrable case of a rotational motion analogous to that of Lagrange and Poisson for a gyrostat in a Newtonian force field, Celest. Mech. Dyn. Astron. 60 (1994) 317-330.

[8] J.E.Cochran, P.H. Shu, S.D. Rew, Attitude motion of asymmetric dual-spin spacecraft, J. Guid. Control Dynam. 5 (1982) 37-42.

[9] A. Deprit, A. Elipe, Complete reduction of the Euler-Poinsot Problem, J. Astronaut. Sci. 41 (1993) 603-628.

[10] A. Elipe, M. Arribas, A. Riaguas, Complete analysis of bifurcations in the axial gyrostat problem, J. Phys. A: Math. and Gen. 30 (1997) 587-601.

[11] A. Elipe, V. Lanchares, Phase flow of an axially symmetrical gyrostat with one constant rotor, J. Math. Phys. 38 (1997) 3533-3544.

[12] A. Elipe, V. Lanchares, Two equivalent problems: Gyrostats in free motion and parametric quadratic Hamiltonians" Mech. Res. Comm. 24 (1997) 583590 .

[13] A. Elipe, V. Lanchares, Exact solution of a triaxial gyrostat with one rotor, Celest. Mech. Dyn. Astron. 101 (2008) 49-68.

[14] M.T. de Bustos Muñoz, J.L. García Guirao, J.A. Vera López, A. Vigueras Campuzano, On Sufficient Conditions of Stability of the Permanent Rotations of a Heavy Triaxial Gyrostat, Qual. Theory Dyn. Syst. 14 (2015) 265280 .

[15] C.D. Hall, Spinup dynamics of biaxialgyrostats, J. Astronaut. Sci. 43 (1995) 263-275. 
[16] C.D. Hall, Spinup dynamics of gyrostats, J. Guid. Control Dynam. 18 (1995) 1177-1183.

[17] C.D. Hall, R.H. Rand, Spinup dynamics of axial dual-spin spacecraft, J. Guid. Control Dynam. 17 (1994) 30-37.

[18] D. Holm, J.E. Marsden, T.S. Ratiu, A. Weinstein, Nonlinear stability of fluid and plasma equilibria, Phys. Rep. 123 (1985) 1-116.

[19] P.C. Hughes, Spacecraft attitude dynamics. John Wiley \& Sons, New York. 1986.

[20] M. Iñarrea, V. Lanchares, Chaos in the reorientation process of a dualspin spacecraft with time dependent moments of inertia, Int. J. Bifurcation Chaos 10 (2000) 997-1018.

[21] T.J. Kalvouridis, Stationary solutions of a small gyrostat in the Newtonian field of two bodies with equal masses. Nonlinear Dyn. 61 (2010) 373-381.

[22] A.M. Kovalev, Stability of steady rotations of a heavy gyrostat about its principal axis, Pmm-J. Appl. Math. Mech. 44 (1981) 709-712.

[23] A.M. Kovalev, Stability of stationary motions of mechanical systems with a rigid body as the basic element, Nonlinear Dynamics and Systems Theory. 1 (2001) 81-96.

[24] V. Lanchares, A. Elipe, Bifurcations in biparametric quadratic potentials, CHAOS: An interdisciplinary journal of nonlinear science 5 (1995) 367-373.

[25] V. Lanchares, A. Elipe, Bifurcations in biparametric quadratic potentials. II, CHAOS: An interdisciplinary journal of nonlinear science 5 (1995) 531535.

[26] V. Lanchares, M. Iñarrea, J.P. Salas, Spin rotor stabilization of a dualspin spacecraft with time dependent moments of inertia, Int. J. Bifurcation Chaos 8 (1998) 609-617.

[27] V. Lanchares, M. Iñarrea, J.P. Salas, J.D. Sierra, A. Elipe, Surfaces of bifurcation in a triparametric quadratic Hamiltonian, Physical Review E 52 (1995) 5540-5548.

[28] E. Leimanis, The General Problem of the Motion of Coupled Rigid Bodies About a Fixed Point, Springer-Verlag, Berlin. 1965.

[29] J.E. Marsden, Lectures on Mechanics, Cambridge University Press, Cambridge, 1992.

[30] J.P. Ortega, T.S. Ratiu, Non-linear stability of singular relative periodic orbits in Hamiltonian systems with symmetry, J. Geom. Phys. 32 (1999) $160-188$. 
[31] V.V Rumiantsev, On the stability of motion of gyrostats, Pmm-J. Appl. Math. Mech. 25 (1961) 9-19.

[32] M.E. Sansaturio, A. Vigueras, Translatory-rotatory motion of a gyrostat in a Newtonian force field, Celest. Mech. 41 (1988) 297-311.

[33] V.A. Sarychev, Dynamics of an axisymmetric gyrostat satellite under the action of a gravitational moment, Cosmic Res. 48 (2010) 18893.

[34] V.A. Sarychev, Dynamics of an axisymmetric gyrostat satellite. Equilibrium positions and their stability, Pmm-J. Appl. Math. Mech. 78 (2014) 249257.

[35] V. Tsogas, T.J. Kalvouridis, A.G. Mavraganis, Equilibrium states of a gyrostat satellite moving in the gravitational field of an annular configuration of $n$ big bodies, Acta Mechanica 175 (2005) 181-195.

[36] J.A. Vera, A. Vigueras, Estabilidad de ciertos equilibrios de un giróstato simétrico bajo un potencial con simetría axial $\mathbf{U}\left(\mathbf{k}_{3}\right)$, in Métodos de dinámica orbital y rotacional (Proc. IV Jornadas de Trabajo en Mecánica Celeste), 175-181. Servicio de Publicaciones Universidad de Murcia, 2002.

[37] J.A. Vera, A. Vigueras, Soluciones de equilibrio en un problema generalizado del de Lagrange-Poisson: condiciones necesarias y suficientes de estabilidad, Monografías de la Real Academia de Ciencias de Zaragoza 22 (2003) 141-150. 